\title{
METACHOMSKIANISMO: ENTRE LA PASIÓN Y EL RECELO
}

Sobre El camino desde Syntactic Structures. Un recorrido bistórico y filosófico por la lingüística chomskiana. Gonzalo, Adriana; Carrió, Cintia y Parera, Griselda (Comp.). Santa Fe: Ediciones UNL, 2013. 271pp.

Cintia Carrió

UNL

CONICET

La obra lingüística de Noam Chomsky ha despertado, desde sus comienzos, tanto grandes pasiones como rechazos manifiestos. Sus postulados teóricos en busca de respuestas a problemas lingüísticos y la arquitectura gramatical supuesta han recibido calificativos que recorren los extremos de un continuum que lo llevan desde la absoluta lucidez hasta el absurdo tecnicismo. Ya sea que se mire esta discusión desde un extremo u otro, de lo que no cabe duda es del impacto que este investigador tuvo y tiene en el modo de pensar el lenguaje, las lenguas y los estudios sobre el lenguaje. En este punto nos vimos tentados a escribir "el lenguaje humano" pero lo fascinante, incluso, es que su obra ha representado un gran avance para la investigación del lenguaje no humano, basta revisar rápidamente las repercusiones que ha tenido y tienen los desarrollos de este teórico en lo que refiere a los estudios sobre el lenguaje animal.

El camino desde Syntactic Structures es un libro que recoge algunas de las discusiones generadas en el marco del I 
Workshop de Historia y Filosofía de la Lingüística Chomskiana en la Facultad de Humanidades y Ciencias de la Universidad Nacional del Litoral (Santa Fe). En dicho marco, un conjunto de especialistas, lingüistas y filósofos, debatieron en torno a diversas temáticas contenidas en la obra lingüística de Noam Chomsky, desde su Syntactic Structures (1957) hasta sus discusiones biolingüísticas enmarcas en las líneas del Minimalist Program (1995 en adelante). A diferencia de las discusiones frecuentes en relación con la obra de Chomsky, las cuales versan sobre temáticas lingüísticas (o biolingüísticas) específicas, en esta obra la mirada se desplaza hacia una arista menos frecuentada: su lectura histórica y metateórica.

Los artículos compilados tienen como común denominador la problemática del cambio teórico y la reconstrucción histórica sistemática. El libro recorre interrogantes que conducen a revisar la idea de continuidad o ruptura que pueda establecerse entre los diferentes momentos de la propuesta chomskiana. La posibilidad de considerar a la propuesta como una teoría constituida por una sucesión de modelos o, desde otra óptica, como un programa de investigación que involucra una sucesión de teorías. Las diferentes posturas que, al respecto, puede encontrar el lector a lo largo del libro lo posicionan en un lugar estratégico, el de tomar conocimiento respecto de la complejidad del problema y, a la vez, considerar la diversidad de miradas sobre la manera de pensarlo. Las opciones epistemológicas divergentes respecto de cómo pensar la reconstrucción de la propuesta chomskiana no sólo resulta manifiesta a lo largo del libro sino que salta a la vista al considera los modos de referirlas de acuerdo a las nominalizaciones seleccionadas (considérese: "teoría", "conjunto de modelos", "programa", entre otras).

El libro se organiza en artículos y comentarios que permiten volver sobre las discusiones de manera que sea posible 
recuperar los diálogos suscitados en función de los distintos temas. En el primero de los artículos, Eguren revisa y explica los cambios que han tenido lugar a lo largo de la lingüística chomskiana en lo que se refiere a la caracterización del contenido de la Gramática Universal (GU). Haciendo foco en el componente sintáctico, recorre una línea que conduce al lector desde una mirada sobrespecificada de la GU hacia la concepción infraespecificada que se desprende de las ideas minimalistas. El comentario de Múgica, por su parte, reacomoda la discusión respecto de la especificación de la GU en función de una tríada de interrogantes que permiten recorrer y reconocer los hitos de la propuesta centrándose en los problemas lingüísticos que aspira a responder.

En la segunda parte del texto, Carrió revisa los vaivenes que se fueron dando en "el programa de investigación chomskiano" de la mano de los primitivos y su vinculación con las adecuaciones descriptiva y explicativa, y los intentos por alcanzar un diseño óptimo para la gramática concluyendo en que la selección de los primitivos se reajusta de acuerdo a una fuerte motivación tanto empírica como teórica. Considera entonces que la tendencia resulta ser aumentar el número de primitivos, disminuir al mínimo las operaciones y especificar los condicionamientos. En su comentario al texto, Palachi se centra en dos problemas: las categorías léxicas en relación con las adecuaciones descriptiva y explicativa por un lado; y, las relaciones entre el léxico y la sintaxis, por otro. Revisa así la tensión entre categoría y función al momento de pensar los primitivos teóricos desde una concepción estructuralista o generativista, lo que le permite mostrar "cómo la construcción de herramientas para la observación del lenguaje por parte de la teoría tiene consecuencias para el trabajo del lingüista en cuanto a la descripción y la explicación" (70). 
Defagó corre el foco de la atención hacia las unidades de estudio de la lingüística y discute las implicancias de la relevancia sobresaliente del tercer factor. Así postula que este hecho tiene consecuencias a nivel cognitivo, dado que induce a rever los procesos mentales implicados en el desarrollo del lenguaje; y, de la misma manera, conduce a revisar las unidades a las que alcanzan estos procesos. La autora sostiene que "no existirían restricciones para que dichos cómputos deban circunscribirse necesariamente y exclusivamente a la organización de los patrones oracionales" (82). A partir de allí, entonces, argumenta a favor de una explicación de las relaciones interoracionales desde los principios de eficiencia computacional.

La tercera parte del libro se ocupa de problemas semánticos, y reúne los artículos de Bassano y Miranda Alonso junto al comentario propuesto por Parera sobre este último texto. Bassano se ocupa del problema de la referencia haciendo hincapié en su estatuto teórico y su lugar en el marco del PM. Para ello organiza su artículo en dos momentos, se dedica inicialmente al tratamiento que se le dio a este problema y sus derivados en la primera versión del PM para luego analizar sus reformulaciones desde el Minimalismo Biolingüístico. Esta discusión le permite concluir en la concepción internalista de la semántica que ve al significado como un conjunto de rasgos semánticos en interface con un sistema mental (conceptualintencional), la semántica está constituida por reglas que mapean representaciones mentales mediante procedimientos bien definidos.

Miranda Alonso comienza repasando los modos en que la propuesta chomskiana, a lo largo de sus diferentes momentos, explica el significado oracional, para luego dedicarse a mirar el problema del significado desde la semántica de la comprensión o semántica cognitiva. En su texto además denuncia una 
actitud de renuncia en la obra chomskiana en relación con las posibilidades de avanzar en la construcción de una teoría semiótica general dada la imposibilidad de constituirse en objeto de un estudio naturalista del lenguaje. Por su parte, Parera dialoga con el texto de Miranda Alonso fundamentalmente en torno a dos ejes. En principio recupera el problema de los límites en la explicación del significado y lo discute en consonancia con los supuestos respecto de la relación entre semántica, aparato sintáctico y conocimiento enciclopédico y estados mentales que organizan el mundo en forma de representaciones. Luego se detiene en el análisis de la propuesta de acercamiento a la semántica de la comprensión y las implicancias supuestas por esta puesta en relación con los postulados chomskianos.

El texto de Skidelsky abre la cuarta parte de este libro e inaugura la discusión que pone en relación a la lingüística chomskiana con la filosofía del lenguaje y de la mente. Skidelsky analiza detalladamente el predominio de la versión epistémica de la discusión sobre la competencia lingüística o la lengua-I por sobre una versión arquitectural asumiendo que esta última es la más adecuada para los desarrollos generativos minimalistas. Expone la versión epistémica postulando que la misma no parece otorgarle inteligibilidad a la idea de competencia lingüística. Así pues, a lo largo del escrito presenta, ejemplifica y discute las razones a favor de la versión epistémica, esto es, la utilización de vocabulario epistémico para la representación de la facultad del lenguaje $(\mathrm{Fl})$, la idea de conocimiento lingüístico tácito, la idea de que la Fl no constituye un mecanismo modular y los argumentos de la pobreza de estímulo a favor de la GU. Este artículo es comentado por Barbieris quien contribuye con dos aportes sustanciales. El primero relativo a la pobreza de estímulo y la subdeterminación de los datos para dar cuenta de la 
inadecuación de las explicaciones empiristas de la adquisición. $\mathrm{Y}$ el segundo relacionado con la posibilidad de concebir la teoría de la $\mathrm{Fl}$ como una teoría de la propiedad, así sostiene que lo que la teoría ofrece es un análisis funcional de la capacidad de integrar recursiva y estructuradamente sonido y significado en términos de los componentes de la Fl.

A partir del texto de Longa, y el comentario que del mismo presenta Blanco, comienzan a pensarse problemas vinculados con el desarrollo de la biolingüística. El artículo de Longa aborda con detalle el desfase entre la filogenia y la ontogenia propiciado por la gramática generativa para lo cual recorre las bases del neodarwinismo a fin de mostrar en qué forma la gramática generativa rechaza dichas bases en relación con la filogenia pero las acepta para la ontogenia. Para cerrar su artículo, Longa muestra una mirada integrada de estos planos, posicionándose desde el PM, perspectiva que le permite acercarse a la propiedad de parsimonia entendida, desde el inicio del artículo, como una de las que caracterizan a las buenas teorías. Blanco recupera la discusión respecto del programa adaptacionista en cómodo paragón con el cuento de Perrault, Caperucita Roja, para discutir sobre la emergencia filogenética del lenguaje y presenta argumentos más que interesantes para revisar este tema.

La parte final de este libro reúne los trabajos de Peris Viñé, el comentario que de él ofrece Audano y el artículo de Gonzalo, ofreciendo así una mirada a la lingüística chomskiana desde la filosofía de la ciencia.

Peris Viñé pone de manifiesto una serie de déficit en la fundamentación de la Corriente Generativa Transformacional (CGT) considerando "déficit" a la adopción de ciertas asunciones sin contar con razones suficientes. Así entonces, revisa lo que llama "posición oficial" respecto de la CGT en 
relación con su naturaleza y alcance. A partir de allí analiza, por ejemplo, la tesis del lenguaje internalizado versus la tesis del lenguaje externalizado y su incidencia en diferentes decisiones y proyecciones. Otros tópicos en los que se centra la atención son: la incomensurabilidad de la CGT con la gramática distribucional, la concepción mentalista del lenguaje y de la gramática como teoría de la mente, la concepción innatista de la adquisición del lenguaje y las pretensiones de naturalización de la lingüística. En su comentario sobre el artículo, Audano recupera algunos de los tópicos presentados por Peris Viñé y recorre la historia de la obra chomskiana desde Chomsky y desde sus principales historiadores.

Por último Gonzalo se ocupa de la problemática de la explicación científica en el marco de la concepción estructural. En función de ello postula la necesidad de referirse en este caso a una teoría macro con cambios modelo-teóricos operados en ella. Estas presentaciones modeloteóricas serían, entonces, susceptibles de representar una red teórica relativa a un tiempo del desarrollo evolutivo de la teoría chomskiana. A su vez, Gonzalo sostiene la necesidad de reconocer los cambios modelo-teóricos de índole intrateórica como cambios parciales. Esta reconstrucción se centra especialmente en la explicación semántica.

Así entonces, este libro conjuga un panorama histórico de la obra chomskiana abarcando discusiones sintácticas, morfológicas y semánticas; $y$, a su vez, incluye material que se ocupa de los vínculos de la lingüística chomskiana con la filosofía del lenguaje, la filosofía de la mente y la filosofía de la ciencia, tanto como con los desarrollos recientes de la biolingüística. 


\section{Bibliografía}

Chomsky, N. (1957). Syntactic Structures. La Haya: Mounton.

Chomsky, N. (1995). The Minimalist Program. Cambridge, Mass.: The MIT Press. 